\title{
MANGROVE MAPPING WITH THE FREEMAN-DURDEN POLARIMETRIC DECOMPOSITION AND INSAR COHERENCE FROM ALOS-2
}

\author{
Tien-Hao Liao ${ }^{1 *}$, Marc Simard $^{2}$, Charlie Marshak ${ }^{2}$, Michael Denbina ${ }^{2}$ and Nathan Thomas ${ }^{3}$ \\ ${ }^{1}$ Division of Geological and Planetary Sciences, California Institute of Technology, CA 91125, USA \\ 2 Jet Propulsion Laboratory, California Institute of Technology, Pasadena, CA 91109, USA \\ ${ }^{3}$ Earth System Science Interdisciplinary Center, UMD/NASA GSFC, College PARK, MD 20771, USA
}

\begin{abstract}
We map mangrove extents in Pongara National Park, Gabon using the Freeman-Durden Decomposition and InSAR Coherence derived from ALOS-2 imagery. Specifically, we obtain a land cover map derived from both this polarimetric decomposition and a 14-day repeat-pass coherence. Our classification model and results are highly interpretable based on a depth 2 decision tree. We further illustrate the correlation between InSAR coherence and height obtaining rough mangrove height estimates from TanDEM-X data. From our results, we observe that repeat-pass interferometric coherence provides invaluable information about mangrove extents and coastal forests. The clear identification of mangrove extents presents a significant opportunity for NISAR, which will provide 12-day repeat pass images over coastal areas globally.
\end{abstract}

Index Terms- Mangrove Extent; Mangrove Height; NISAR; L-band SAR; Microwave Remote Sensing.

\section{INTRODUCTION}

Mangroves forests serve as a buffer for terrestrial life and provide a rich and diverse coastal habitat in tropical and subtropical zones [1]. Moreover, they offer a significant source of global carbon storage [1]. As such, monitoring the extent and health of mangrove forests is an important application area for L-band SAR [1,2], which is able to penetrate both the mangrove canopy and the clouds that are omnipresent in tropical and subtropical zones.

Global mangrove extents have been mapped at the 25 meter scale with ALOS/PALSAR backscatter and Landsat in the Global Mangrove Watch data products [1,2]. Our aim is to investigate additional possible approaches utilizing polarimetric and interferometric data that will be available in coastal regions with NISAR [3] to produce such mangrove maps. We are motivated in part by the numerous studies utilizing the

This research was carried out at the Jet Propulsion Laboratory, California Institute of Technology, under a contract with the National Aeronautics and Space Administration. (c)2019. California Institute of Technology. Government sponsorship acknowledged
Freeman-Durden decomposition to map mangrove forests regionally such as in $[4,5]$. The future NISAR misssion will provide SAR images of dual-pol(HH and HV) or up to quadpol. The revisiting cycle is further shortened to 12 days enabling the monitoring of coastal regions using coherence also in every 12 days. There have been numerous studies to relate repeat-pass coherence to tree height as in $[6,7]$. Indeed, InSAR coherence and polarimetric decompositions of tropical forests are impacted by environmental factors such as rain, wind, and flooding [8]. Thus, we cannot expect a model to be trained using one interferometric pair at one particular site to be valid for another pair at that site or elsewhere. However, our results suggest that mangrove areas are so clearly differentiated within such L-band imagery that we expect larger studies can leverage an unsupervised approach regionally.

Using our mangrove extents, we estimate tree height from $\mathrm{HH}$ coherence to further highlight the correlative relationship between repeat pass InSAR coherence and canopy height. We use TanDEM-X elevation above mean sea level as a proxy for mangrove height as in [9]. Then, we train a linear model to estimate tree height using sparse TanDEM-X data to obtain our mangrove height estimates.

\section{DATA AND METHODOLOGY}

In this study, we obtain a land cover classification from ALOS-2 polarimetric decomposition and InSAR coherence data using a depth-2 decision tree [10]. We then estimate mangrove height to emphasize the correlative relationship between mangrove height and InSAR coherence.

Our classification model uses three ALOS-2 products: the 14-day repeat pass $\mathrm{HH}$ coherence, and the volumetric and double-bounce scattering components of the FreemanDurden decomposition. We obtained the HH coherence using ISCE-2 [11] and the Freeman-Durden decomposition [12] using PolSARPro [13]. We also used a Landsat-8 landcover reference map generated by Thomas et al (unpublished) using a cloud free image of the park. Landsat- 8 imagery was segmented into image objects and classified using a random forest algorithm within RSGISLib [14]. The land cover ref- 


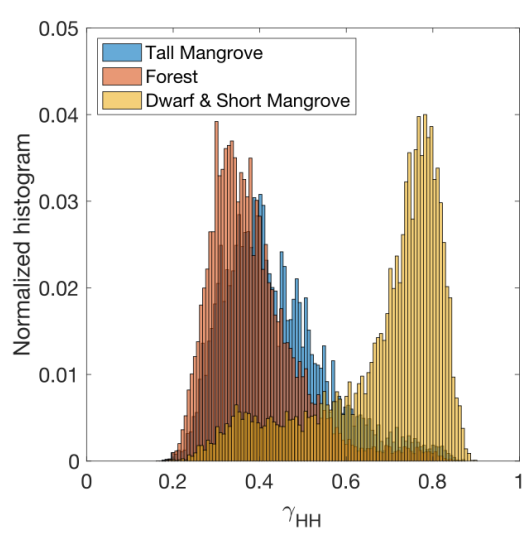

(a)

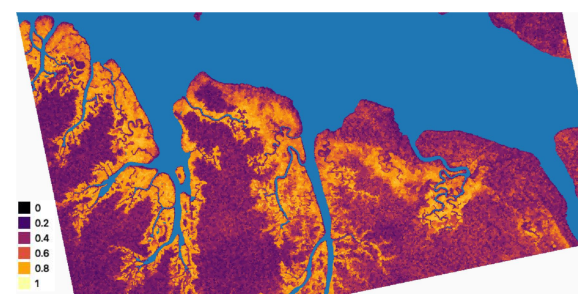

(d)

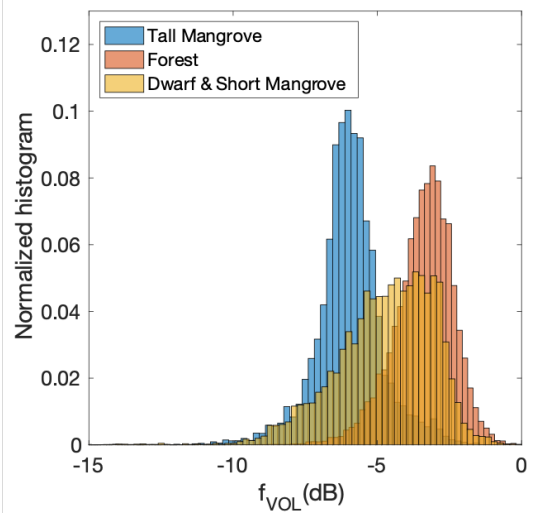

(b)

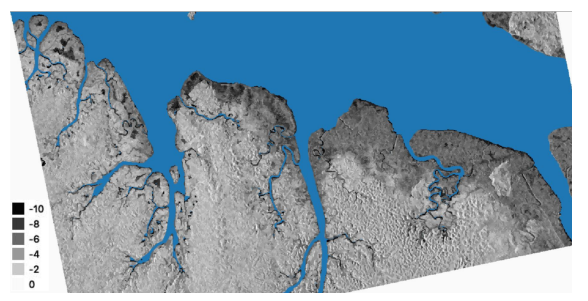

(e)

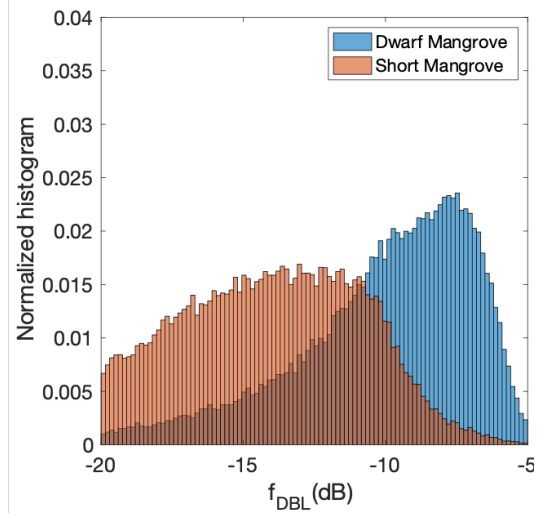

(c)

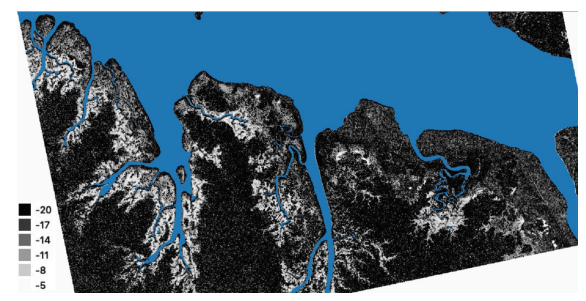

(f)

Fig. 1: Top Row: histograms of pixels by class displaying (a) HH coherence, (b) volumetric scattering, and (c) double bounce scattering. Bottom Row: maps of (d) HH coherence, (e) volumetric scattering, and (f) double bounce scattering.

erence map identifies mangroves (dwarf, short, and tall), forest, water, bare ground, and urban areas. For our study we only consider mangrove and forest classes assuming a crude canopy cover map will be available globally. Before beginning the classification, we performed an initial histogram analysis of the ALOS-2 products shown in the top row of Figure 1. We observed that the $\mathrm{HH}$ coherence is sensitive to canopy height and should be able to differentiate between (a) short/dwarf mangroves and (b) tall mangroves/forest. Additionally, volume scattering is able to differentiate between (a) tall mangrove and (b) forest while double bounce can differentiate between (a) dwarf mangroves and (b) short mangroves.

Because the histogram analysis showed clear differentiation between forest and mangrove classes, we use a decision tree to automatically determine the thresholds for determining such a classification [10]. We trained our decision tree using 500 random pixels from each mangrove and forest class. We use the depth 2 tree so the model can be more easily explained and analyzed. Our final map is generated using a majority filter to correct (a) forest pixels misclassified as mangroves due to the shadow of the canopy seen in the volumetric scattering and (b) tall mangroves adjacent to the rivers which have higher double bounce signal than those tall mangroves farther inland.

To emphasize the relationship between coherence and height we perform a crude height estimate of mangroves from $\mathrm{HH}$ coherence. We observed a monotonically decreasing relationship between coherence and mangrove height, the latter being estimated from TanDEM-X as in [9]. We utilize a standard unbiased linear model to retrieve mangrove heights [15].

\section{RESULTS}

Using the model described in Section 2, we retrieve mangrove and forest classes in Figure 2. Our model is described by the rules shown in Figure 3. Our model determines these thresholds automatically from the training data [15]. As we noted earlier, the $\mathrm{HH}$ coherence is examined at the first node and differentiates between short/dwarf mangroves (low canopy height) and forest/tall mangroves (large canopy height). A confusion matrix is shown in Table 1 and the accuracy metrics by class are shown in Table 2 . Using our approach, we are able to differentiate between forest and mangroves with over $90 \%$ producer/user accuracy as illustrated in Table 2 indicating that our method will be valuable for mapping mangrove extents. Indeed, short and dwarf mangroves have the lowest per class accuracy because (a) coherence is less sensitive at lower heights and (b) double bounce is more sensitive to speckle noise than the other polarimetric components.

We estimate mangrove height using $\mathrm{HH}$ coherence to further highlight the relationship between repeat-pass coherence 


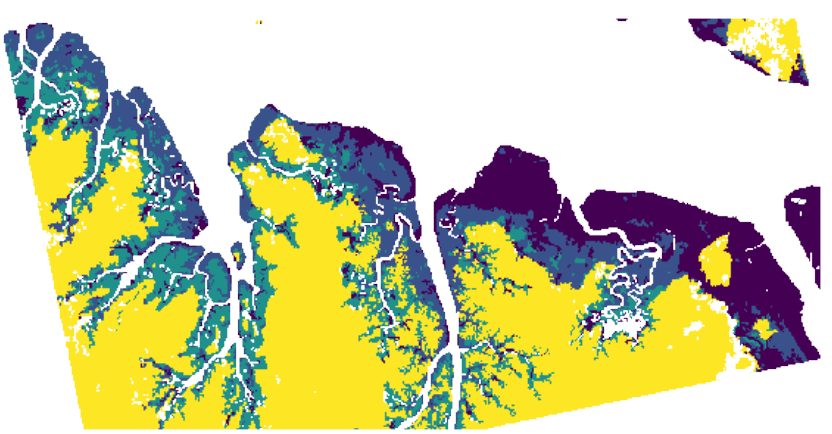

(a) Reference

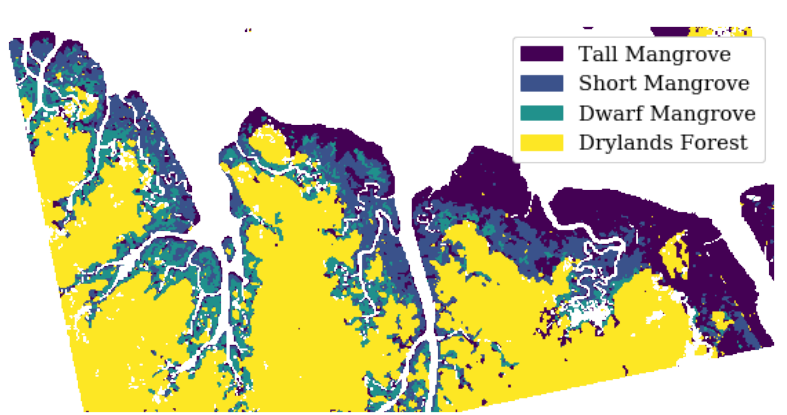

(b) Retrieved

Fig. 2: Reference and retrieved land cover maps.

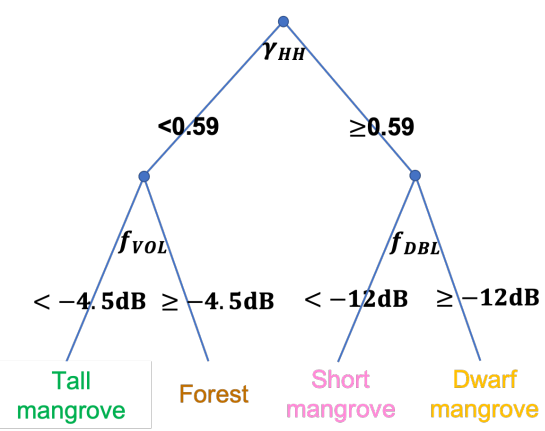

Fig. 3: Rules for the decision tree for our land cover classifier.

and height. We train our model again on just 500 points randomly sampled from each class. The height estimate is rough having 10 meter RMSE ( $86 \%$ normalized by the mean height). However, we have an $R^{2}$ of .39 of per pixel height estimates with the TanDEM-X height product. In Figure 4, we plot 3-meter binned TanDEM-X height compared with the same height estimate. We see from the binned comparison that the model saturates at approximately 20 meters.

\section{CONCLUSIONS}

Using the Freeman-Durden decomposition and InSAR coherence derived from ALOS-2, we classify mangrove and forested areas using a decision tree. We validate our classification with a Landsat- 8 derived landcover map. We show that the boundary between mangrove and forested areas are highly differentiated indicating such L-band products will be invaluable for NISAR mangrove monitoring. The model discussed here is easily interpreted and will serve as a reference for future classification models to map mangroves from L-band imagery. In future work, we will explore more sophisticated classification methodologies to automate this classification process over numerous sites to further illustrate NISAR mangrove mapping capabilities.

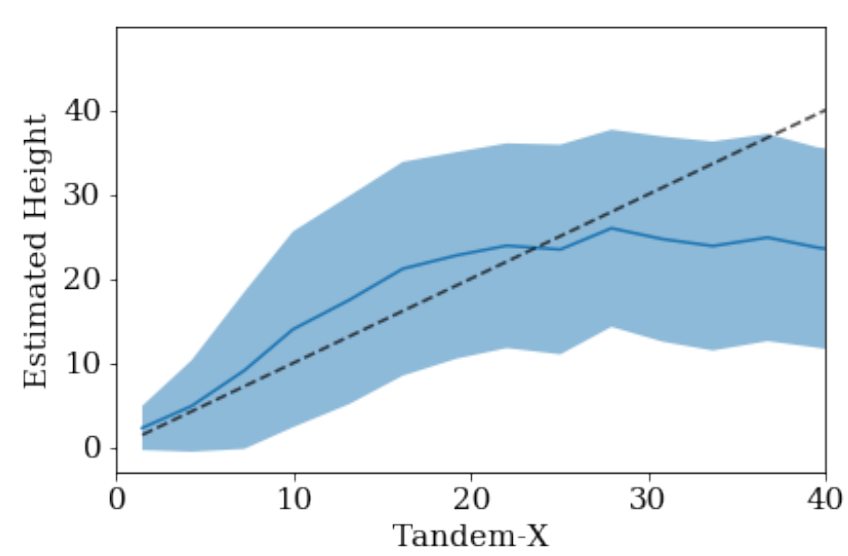

Fig. 4: Estimated height vs. TanDEM-X height using 3 meter bins of TanDEM-X product. Black dashed line is $y=x$ and fill is one standard deviation of estimates within 3 meter TanDEM-X bin.

\section{REFERENCES}

[1] P. Bunting, A. Rosenqvist, R. Lucas, L.-M. Rebelo, L. Hilarides, N. Thomas, A. Hardy, T. Itoh, M. Shimada, and C. Finlayson, "The Global Mangrove Watch-A New 2010 Global Baseline of Mangrove Extent," Remote Sensing, vol. 10, no. 10, p. 1669, Oct 2018. [Online]. Available: http://dx.doi.org/10.3390/ rs 10101669

[2] N. Thomas, R. Lucas, P. Bunting, A. Hardy, A. Rosenqvist, and M. Simard, "Distribution and Drivers of Global Mangrove Forest Change, 1996-2010," PloS one, vol. 12, no. 6, p. e0179302, 2017.

[3] NISAR Science Team, "NASA-ISRO SAR Mission Science Users' Handbook," https://nisar.jpl.nasa.gov/files/ nisar/NISAR_Science_Users_Handbook.pdf, 2019, [Online; accessed January-2019]. 


\begin{tabular}{lrrrr}
\hline $\begin{array}{l}\text { Predicted } \\
\text { Reference }\end{array}$ & Drylands Forest & Dwarf Mangrove & Short Mangrove & Tall Mangrove \\
\hline Drylands Forest & 249913 & 2526 & 5056 & 6485 \\
Dwarf Mangrove & 19651 & 46529 & 17261 & 3857 \\
Short Mangrove & 5168 & 9525 & 56349 & 21030 \\
Tall Mangrove & 4402 & 2343 & 6476 & 76008 \\
\hline
\end{tabular}

Table 1: The confusion matrix using Landsat-8 classifcation reference.

\begin{tabular}{lrr}
\hline & Producer & User \\
\hline Drylands Forest & 0.95 & 0.90 \\
Dwarf Mangrove & 0.53 & 0.76 \\
Short Mangrove & 0.61 & 0.66 \\
Tall Mangrove & 0.85 & 0.71 \\
\hline Total Accuracy & \multicolumn{2}{c}{0.8051} \\
Cohen's $\kappa$ & 0.7048 \\
\hline
\end{tabular}

Table 2: Classification metrics for retrieved land cover.

[4] C. Proisy, E. Mougin, F. Fromard, and M. Karam, "Interpretation of Polarimetric Radar Signatures of Mangrove Forests," Remote Sensing of Environment, vol. 71, no. 1, pp. 56-66, 2000.

[5] I. Brown, S. Mwansasu, and L.-O. Westerberg, "L-Band Polarimetric Target Decomposition of Mangroves of the Rufiji Delta, Tanzania," Remote Sensing, vol. 8, no. 2, p. 140, 2016.

[6] M. Simard and M. Denbina, "An Assessment of Temporal Decorrelation Compensation Methods for Forest Canopy Height Estimation using Airborne L-band Same-day Repeat-pass Polarimetric SAR Interferometry," IEEE Journal of Selected Topics in Applied Earth Observations and Remote Sensing, vol. 11, no. 1, pp. 95-111, 2017.

[7] M. Lavalle, M. Simard, and S. Hensley, "A Temporal Decorrelation Model for Polarimetric Radar Interferometers," IEEE Transactions on Geoscience and Remote Sensing, vol. 50, no. 7, pp. 2880-2888, 2011.

[8] C. N. Koyama, M. Watanabe, M. Hayashi, T. Ogawa, and M. Shimada, "Mapping the Spatial-temporal Variability of Tropical Forests by ALOS-2 L-band SAR Big Data Analysis," Remote Sensing of Environment, vol. 233, p. 111372, 2019.

[9] M. Simard, L. Fatoyinbo, C. Smetanka, V. H. Rivera-Monroy, E. Castañeda-Moya, N. Thomas, and T. Van der Stocken, "Mangrove Canopy Height Globally related to Precipitation, Temperature and Cyclone Frequency," Nature Geoscience, vol. 12, no. 1, pp. 40-45, 2019. [Online]. Available: https: //doi.org/10.1038/s41561-018-0279-1

[10] L. Breiman, Classification and Regression Trees. Routledge, 2017.

[11] P. Rosen, E. Gurrola, P. S. Agram, G. F. Sacco, and M. Lavalle, "The InSAR Scientific Computing Environment (ISCE): A Python Framework for Earth Science," in AGU Fall Meeting Abstracts, 2015.

[12] A. Freeman and S. L. Durden, "A Three-component Scattering Model for Polarimetric SAR Data," IEEE Transactions on Geoscience and Remote Sensing, vol. 36, no. 3, pp. 963-973, 1998.

[13] E. Pottier, L. Ferro-Famil, M. Fitrzyk, and Y.-L. Desnos, "PolSARpro-BIO: The New Scientific Toolbox for ESA \& Third Party fully Polarimetric SAR Missions," in EUSAR 2018; 12th European Conference on Synthetic Aperture Radar. VDE, 2018, pp. 1-4.

[14] D. Clewley, P. Bunting, J. Shepherd, S. Gillingham, N. Flood, J. Dymond, R. Lucas, J. Armston, and M. Moghaddam, "A Python-based Open Source System for Geographic Object-based Image Analysis (GEOBIA) Utilizing Raster Attribute Tables," Remote Sensing, vol. 6, no. 7, pp. 6111-6135, 2014.

[15] K. P. Murphy, Machine Learning: A Probabilistic Perspective. MIT Press, 2012. 